\title{
MODELING THE EFFECT OF VEGETATION ON RIVER FLOODPLAIN HYDRAULICS
}

\section{A.I. STAMOU* \\ G. PAPADONIKOLAKI \\ A. GKESOULI \\ A. NIKOLETOPOULOS}

Received: $10 / 05 / 12$

Accepted: 27/07/12

\author{
School of Civil Engineering \\ Water Resources and Environmental Engineering \\ National Technical University of Athens \\ Iroon Polytechniou 5, 15780 Athens, Greece
}

*to whom all correspondence should be addressed: e-mail: stamou@central.ntua.gr

\begin{abstract}
Vegetation in river floodplains has significant influence on the flood hydraulics and fate of suspended sediments, nutrients and contaminants. In the present, work preliminary 3-D calculations were performed to examine the effect of vegetation on the mean flow in open channels using the CFD model CFX-12.1, employing the RANS k-epsilon turbulence model. Calculated flow velocity distributions were compared against an experiment of free surface uniform flow in a vegetated experimental channel, filled with cylindrical submerged elements representing vegetation; these elements were rigid and arranged in a staggered pattern.

Four unstructured numerical grids were employed, ranging from approximately 9.5 to 27.5 millions of tetrahedral elements. The main characteristics of the flow were (a) the formation of small recirculation regions in the wakes of the cylinders and (b) the relative uniform flow conditions throughout the length of the channel. Low flow velocities were observed in the vegetated region, implying the resistance due to vegetation, and higher velocities close to the free surface. The best agreement with experimental data was achieved for the finest grid that also included grid refinement at the top of the cylinders. Grid independence behaviour using relatively very fine grids was rather surprising and requires further detailed investigation.
\end{abstract}

KEYWORDS: Vegetation; eco-hydraulics; hydrodynamic models; turbulence models; fluvial hydraulics; river floodplains.

\section{INTRODUCTION}

Vegetation is one of the major factors that affect the mean and turbulent flow in open channels and the transport processes of suspended sediments and contaminants, thus determining the functioning of wetlands, such as river floodplains. In vegetated surface water bodies the resistance to flow is increasing and the mean flow velocities are reducing, relative to non-vegetated regions, due to the presence of aquatic plants. The interaction between the type of vegetation and the characteristics of flow field is of great interest for the design of flood defense systems and can be examined with the use of mathematical models.

Many studies have been recently carried out on this topic. Klopstra et al. (1997) and Meijer and Van Velzen (1999) developed a method to analytically determine the velocity profile that divides the flow domain into two layers, one within the vegetation and another above it. The model was based on the solution of the momentum equation in the vegetation layer while keeping a logarithmic profile in the upper (two-layer model). Lopez and Garcia (2001) developed a model to compute the mean velocity profiles and turbulence characteristics in open channel vegetated flows. Their model was based on a different approach, relative to Klopstra et al. (1997), since the drag due to vegetation is taken into account not only in the momentum equation, but also in the equations of a modified k-epsilon model; both models were originally developed for submerged vegetation but they can be extended to emergent vegetation. Defina and Bixio (2005) modified the two above-mentioned models to consider 
plant geometry and drag coefficient with depth; also, they added a turbulent kinetic energy budget equation to the two-layer model. Wilson et al. (2006) used the 3D finite volume program SSIIM to model the hydraulic impact of willow stands on the velocity distribution; the model solves the continuity and momentum equations for each cell and employs the k-epsilon model for the modeling of turbulence. The additional hydraulic resistance of the willow stands was modelled separately to the bed resistance using a drag force term that was introduced into the momentum equations.

In the present work preliminary 3-D calculations are performed to examine the influence of vegetation on the mean flow in open channels using the CFD (Computational Fluid Dynamics) model CFX-12.1 (http://www.ANSYS.com) that employs the RANS k-epsilon turbulence model; the results of the model are compared with experiments.

\section{EXPERIMENTAL DATA}

In this work model computations are compared against the experimental data of Exp.12 that were obtained by Dunn et al. (1996), who simulated the free surface uniform flow in a vegetated experimental channel; more computations can be found in Papadonikolaki (2011). The experimental setup was a $19.5 \mathrm{~m}$ long, $0.91 \mathrm{~m}$ wide and $0.61 \mathrm{~m}$ deep tilting flume, filled with cylindrical submerged elements representing vegetation; these elements were rigid and were arranged in a staggered pattern, as shown in Figure 1. The three components of velocity were measured using the threediamensional Acoustic Doppler Velocimeter that also allowed the measurement of velocity fluctuations, Reynolds stresses and turbulence intensity. Average velocity measurements were determined in a centered area of the channel (to avoid effects from the sidewalls) and at least $1.5 \mathrm{~m}$ downstream the first row of the cylinders (to ensure uniform form conditions independent on the inlet conditions); the central measurement area covered a length equal to $1.4 \mathrm{~m}$ and width equal to $0.16 \mathrm{~m}$.

The characteristics of Exp.12 are the following: discharge $=0.181 \mathrm{~m}^{3} \mathrm{~s}^{-1}$, average bed slope $=0.011$, water flow depth $\mathrm{H}=0.233 \mathrm{~m}$, Froude number $=0.58$, diameter of cylinders $=0.00635 \mathrm{~m}$, average height of cylinders $\mathrm{hp}=0.118 \mathrm{~m}$, and cylinder density $=0.62 \mathrm{~m}^{-1}$, that is defined as the ratio between the diameter of the cylinders and the squared distance between two cylinders.

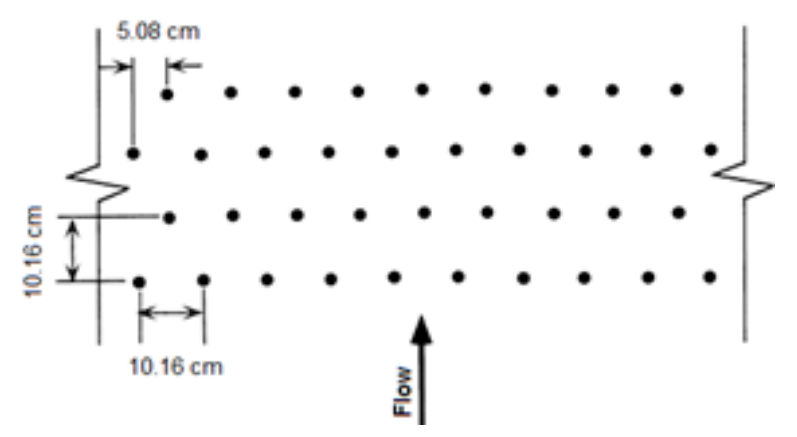

Figure 1. Staggered cylinder pattern (Dunn et al., 1996)

From the available 12 experimental series by Dunn et al. (1996) Exp.12 was chosen to be modeled due to its relatively low vegetation density $\left(0.62 \mathrm{~m}^{-1}\right)$ compared with that of the other experiments $\left(1.09-2.46 \mathrm{~m}^{-1}\right)$; this vegetation density permitted the investigation of the use of various grid sizes.

\section{THE CFD MODEL}

The flow field equations governing the 3-D flow in an open channel are the following:

Continuity equation

$$
\frac{\partial \rho U_{j}}{\partial x_{j}}=0
$$


Momentum equations

$$
\frac{\partial \rho U_{i}}{\partial t}+\frac{\partial \rho U_{j} U_{i}}{\partial x_{j}}=-\frac{\partial P}{\partial x_{i}}+\frac{\partial}{\partial x_{j}}\left(\mu \frac{\partial U_{i}}{\partial x_{j}}-\rho \overline{u_{i} u_{j}}\right)+g_{i} \rho
$$

where $t$ is the time, $x_{i}$ and $x_{j}$ are the Cartesian coordinates in i and j direction respectively, $U_{i}$ and $U_{j}$ are the flow velocities in $i$ and $j$ direction respectively, $\rho$ is the density of water, $P$ is the pressure, $\mu$ is the molecular viscosity of the water and $g_{i}$ is the acceleration of gravity.

For the calculation of the Reynolds (turbulent) stresses $-\rho \overline{u_{i} u_{j}}$ the assumption of the isotropic turbulence is applied combined with the Bussinesq approximation, i.e.

$-\rho \overline{u_{i} u_{j}}=\mu_{t}\left(\frac{\partial U_{i}}{\partial x_{j}}+\frac{\partial U_{j}}{\partial x_{i}}\right)-\frac{2}{3} \rho k \delta_{i j}$

where $\mu_{t}$ is the eddy viscosity, $\delta_{i j}$ is the Kronecker delta $\left(\delta_{i j}=1\right.$ for $i=j$ and $\delta_{i j}=0$ for $\left.i \neq j\right)$ and $k$ is the average turbulent kinetic energy per unit mass, given by

$k=\frac{1}{2}\left(\overline{u_{1}^{2}}+\overline{u_{2}^{2}}+\overline{u_{3}^{2}}\right)$

where $\mathrm{u}_{1}, \mathrm{u}_{2}$ and $\mathrm{u}_{3}$ are the velocity fluctuations in $\mathrm{i}, \mathrm{j}$ and $\mathrm{k}$ direction respectively.

The distribution of $\mu_{t}$ is being determined with the standard $k-\varepsilon$ turbulence model, which relates $\mu_{t}$ to $\mathrm{k}$ and the rate of its dissipation $(\varepsilon)$ via

$\mu_{t}=\rho c_{\mu} \frac{k^{2}}{\varepsilon}$

where $c_{\mu}$ is an empirical constant and $\varepsilon$ is given by the following equation

$\varepsilon=\frac{\mu}{\rho} \frac{\partial u_{i}}{\partial x_{j}} \frac{\partial u_{i}}{\partial x_{j}}$

The distributions of $k$ and $\varepsilon$ are calculated from the following semi-empirical modelled transport equations

$$
\begin{aligned}
& \frac{\partial \rho k}{\partial t}+\frac{\partial \rho U_{j} k}{\partial x_{j}}=\frac{\partial}{\partial x_{j}}\left(\frac{\mu_{t}}{\sigma_{k}} \frac{\partial k}{\partial x_{j}}\right)+G-\rho \varepsilon \\
& \frac{\partial \rho \varepsilon}{\partial t}+\frac{\partial \rho U_{j} \varepsilon}{\partial x_{j}}=\frac{\partial}{\partial x_{j}}\left(\frac{\mu_{t}}{\sigma_{\varepsilon}} \frac{\partial \varepsilon}{\partial x_{j}}\right)+c_{1} \frac{\varepsilon}{k} G-\rho_{C_{2}} \frac{\varepsilon^{2}}{k}
\end{aligned}
$$

where $\mathrm{G}$ is the production term of $\mathrm{k}$ by the mean velocity gradients given as follows

$$
G=\mu_{t}\left(\frac{\partial U_{i}}{\partial x_{j}}+\frac{\partial U_{j}}{\partial x_{i}}\right) \frac{\partial U_{i}}{\partial x_{j}}
$$

The standard values of the constants $c_{\mu}=0.09, c_{1}=1.44, c_{2}=1.92$ and of the turbulent Schmidt numbers $\sigma_{\mathrm{k}}=1.0$ and $\sigma_{\varepsilon}=1.3$ are used in the present computations. 


\section{NUMERICAL DETAILS}

\section{The numerical code}

Calculations were performed with the numerical code CFX-12.1 (http://www.ANSYS.com) that uses the finite volume method for the spatial discretisation of the domain. The equations of the model presented in section 3 are integrated over each control volume, such that the relevant quantity (mass, momentum, $\mathrm{k}$ and epsilon) is conserved, in a discrete sense, for each control volume. For the continuity equation (pressure-velocity coupling), a second order central difference approximation is used, modified by a fourth order derivative in pressure, which redistributes the influence of pressure. The second order upwind Euler scheme approximates the transient term. A scalable and fully implicit coupled solver is used for the solution of the equations.

\section{Boundary conditions}

Boundary conditions are defined at the borders of the computation domain; due to the relatively high length of the experimental flume $(19.5 \mathrm{~m})$ and the very small size of the vegetation elements (diameter of cylinders $=0.00635 \mathrm{~m}$ ) the geometry of the simulation needed to be simplified. After a significant number of preliminary tests it was decided to model a part of the experimental channel having a length equal to $2.0 \mathrm{~m}$; for more details see Papadonikolaki (2011).

To ensure uniform conditions throughout the flume a periodic boundary condition was inserted to the computational domain that provided an interface between the inlet and outlet faces (translational periodicity). A conservative mass flow rate was defined equal to $181 \mathrm{~kg} \mathrm{~s}^{-1}$. The free surface was treated as a free slip wall, using the rigid-lid approximation. Finally, at the walls (bed and sides), which were considered to be smooth, the standard wall function approach was applied, which relates the shear stress at the wall to the cell node velocity component parallel to the wall. The boundary conditions and the three dimensional simplified geometry are shown in Figure 2.

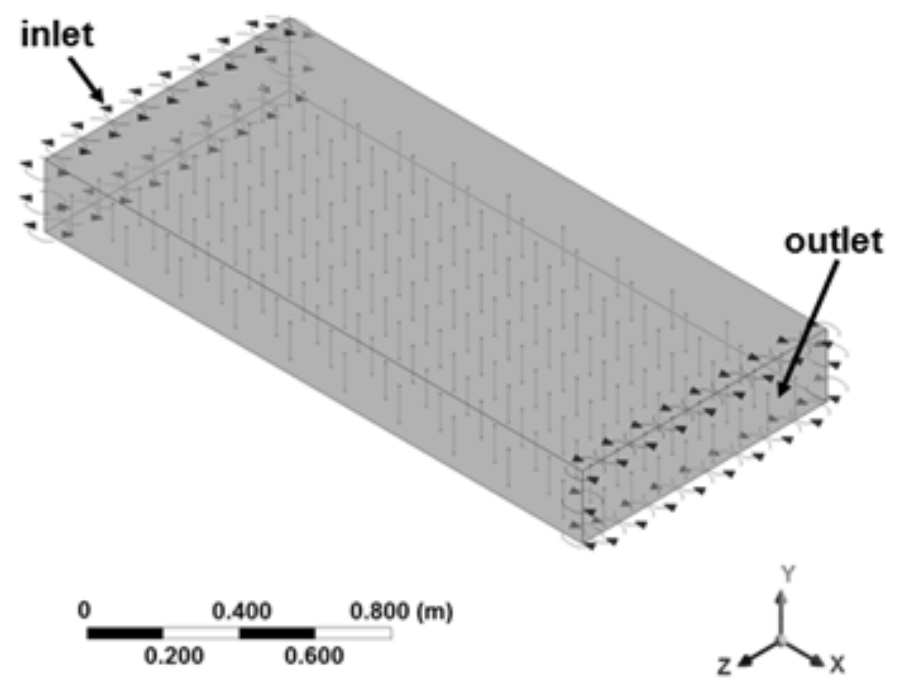

Figure 2. Simplified geometry of the computational domain

\section{Numerical grids and grid independence}

The numerical code employs unstructured numerical grids that permit a very accurate representation of the boundaries. In the present study four numerical grids, which were noted as Grid1 to Grid4, were employed for the simulation of Exp.12 with the characteristics that are shown in Table 1.

The small dimensions of the vegetation elements (cylinders) resulted in sizes of computational grids that ranged from approximately 9.5 to 27.5 millions of tetrahedral elements; finer grids could not be constructed due to the "prohibited" required large size of the computational memory. Grid2 and Grid4 involve a refinement at the top of the cylinders in an attempt to capture small variations of the turbulence kinetic energy and shear stresses in this region. Views of a part of Grid1 (no refinement) and Grid2 (with refinement) are shown in Figure 3. 
Table 1. Characteristics of the numerical grids

\begin{tabular}{|l|c|c|c|c|c|c|}
\hline Grid & $\begin{array}{c}\text { Refine-ment } \\
\text { at the top of } \\
\text { the } \\
\text { cylinders }\end{array}$ & $\begin{array}{c}\text { Min } \\
\text { face } \\
\text { size } \\
\text { (m) }\end{array}$ & $\begin{array}{c}\text { Max } \\
\text { face } \\
\text { size } \\
\mathbf{( m )}\end{array}$ & $\begin{array}{c}\text { Number of } \\
\text { tetrahedral } \\
\text { elements }\end{array}$ & $\begin{array}{c}\text { Number of } \\
\text { nodes }\end{array}$ & $\begin{array}{c}\text { Construction } \\
\text { time } \\
\text { (min) }\end{array}$ \\
\hline Grid1 & No & 0.002 & 0.05 & 9540857 & 1675273 & 20 \\
\hline Grid2 & Yes & 0.002 & 0.05 & 10066821 & 1772777 & 25 \\
\hline Grid3 & No & 0.001 & 0.05 & 26072101 & 4623295 & 40 \\
\hline Grid4 & Yes & 0.001 & 0.05 & 27462598 & 4857062 & 60 \\
\hline
\end{tabular}

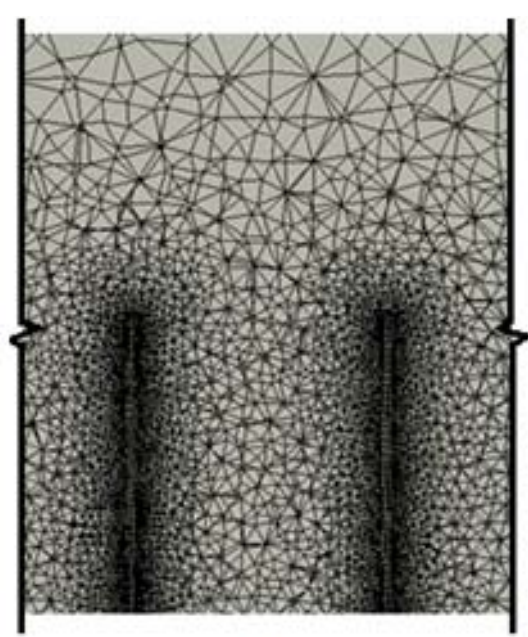

(a)

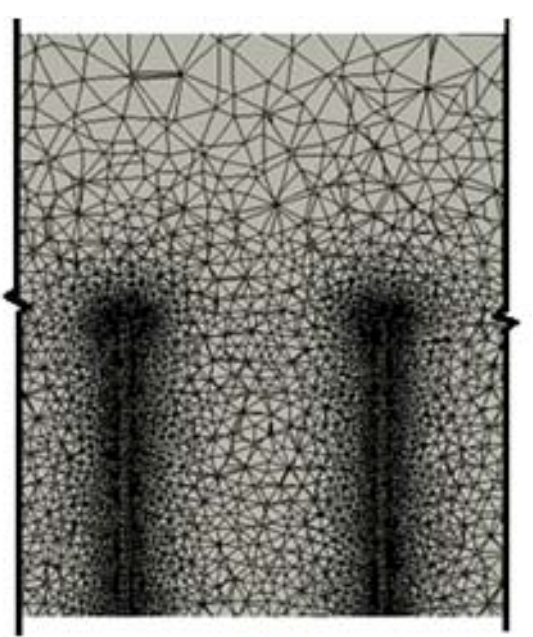

(b)

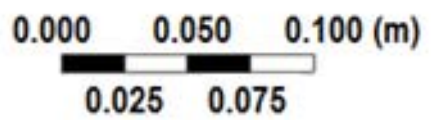

Figure 3. Part of (a) Grid1 (no refinement) and (b) Grid2 (with refinement)

\section{RESULTS AND DISCUSSION}

Figure 4 illustrates the calculated velocity field for Grid4. The main characteristics of the flow are (a) the formation of small re-circulation regions in the wakes of the cylinders; see detail (a) of Figure 4, and (b) the relative uniform flow conditions throughout the length of the channel. The streamlines around a cylinder are also shown in detail (b) of Figure 4.

Thirty five velocity profiles were determined within the central area of the channel including the areas of the wakes of some cylinders; then, average values were calculated and are shown in Figure 5 together with experimental values. Figure 5 depicts that flow velocities are low within the area of vegetation and increase in the layer above the top of the cylinders; large gradients in the velocity distributions are observed at the tops of the cylinders indicating that shear stresses are high in this region. The agreement between computed and measured values within the area of vegetation is satisfactory for the finest mesh (27.5 millions tetrahedral elements) with refinement at the tops of the cylinders (Grid4).

In these preliminary computations grid independent velocity distributions were not achieved, even after using 27.5 millions tetrahedral elements; results for the four numerical grids were notably different (see Figure 5). 


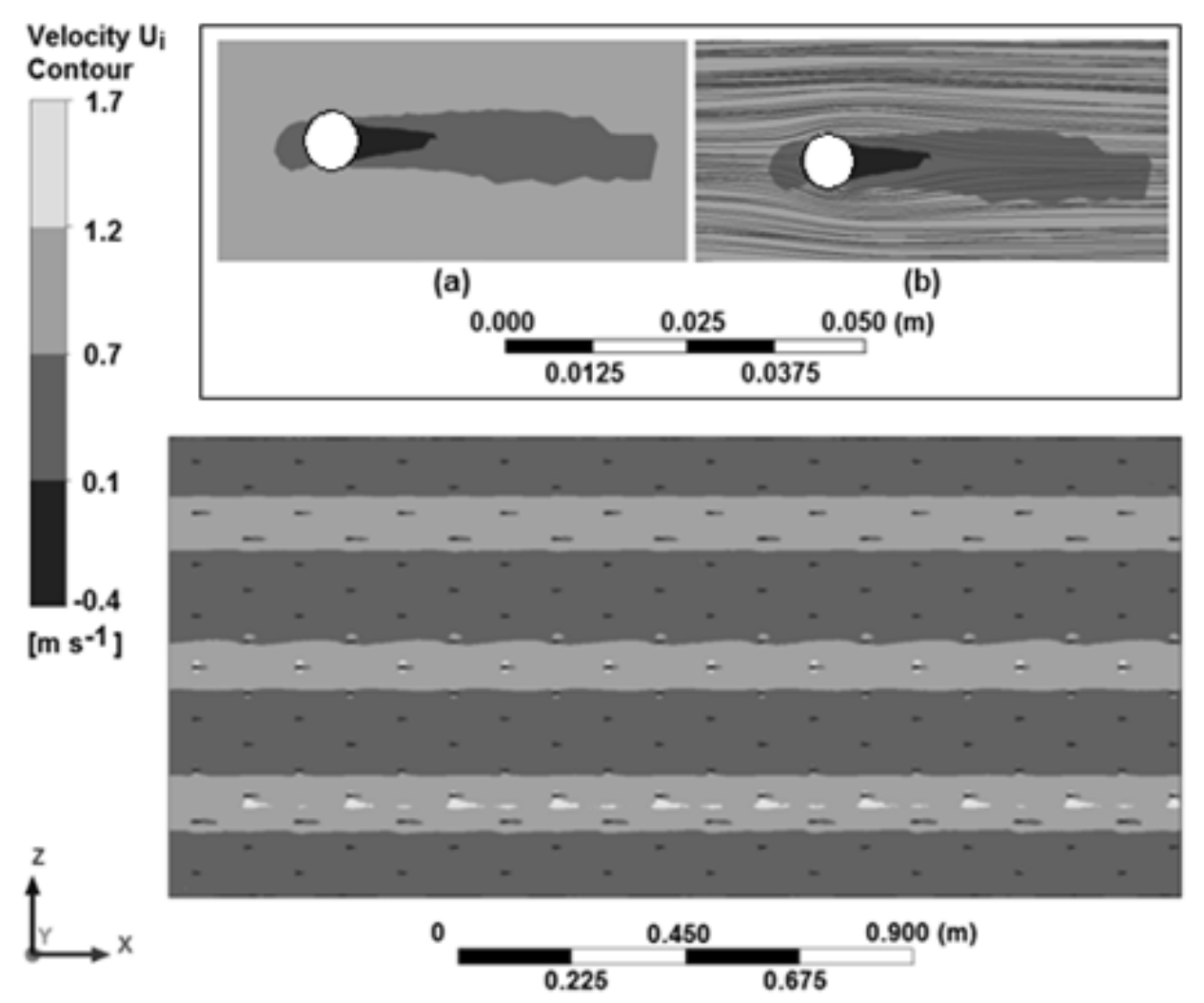

Figure 4. Top view of velocity contours and details of the flow field

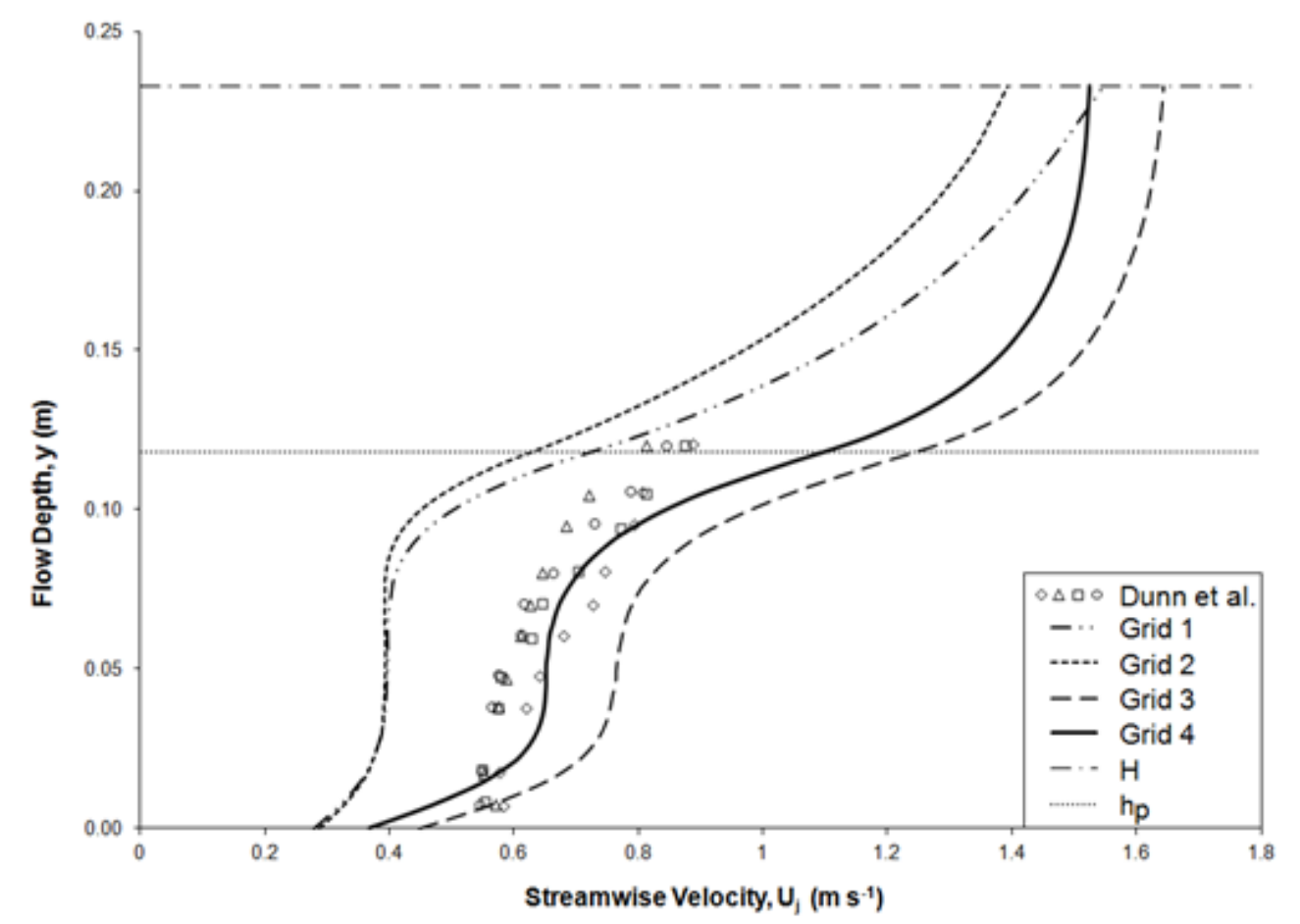

Figure 5.Comparison between calculated velocity distributions and experimental data

\section{CONCLUSIONS AND PROPOSED FUTURE WORK}

Preliminary computations using CFX-12.1 were performed in vegetated channels and calculated velocity distributions were compared to experimental data (Dunn et al., 1996). The main characteristics of the flow are (a) the formation of small re-circulation regions in the wakes of the 
cylinders and (b) the relative uniform flow conditions throughout the length of the channel. Low flow velocities were observed in the vegetated region, which implies the resistance due to the presence of vegetation, and higher velocities close to the free surface.

Calculations with four different grid sizes ranging from 9.5 to 27.5 millions of tetrahedral elements were performed and the best agreement of computations with experiments was achieved for the finest grid that also included grid refinement at the top of the cylinders. This grid independence behaviour using relatively very fine grids was rather surprising and requires further detailed investigation.

\section{ACKNOWLEDGEMENT}

The present work was performed within the Program for Basic Research (PEVE-2010) of the National Technical University of Athens entitled "Computational research of the influence of the vegetation on the flow during floods events".

\section{REFERENCES}

1. ANSYS-CFX, Release 12.1, URL: http://www.ANSYS.com

2. Defina A. and Bixio A.C. (2005), Mean flow and turbulence in vegetated open channel flow, Water Resources Research, 41, 1-12.

3. Dunn C., Lopez F. and Garcia M. (1996), Mean flow and turbulence in a laboratory channel with simulated vegetation, Hydrosystems Laboratory, Department of Civil Engineering, University of Illinois at Urbana-Champaign.

4. Klopstra D., Barneveld H.J., Van Noortwijk J.M. and Van Velzen E.H. (1997), Analytical model for hydraulic roughness of submerged vegetation, Managing Water: Coping With Scarcity and Abundance, 27th IAHR Congress, August 10-15 1997 San Francisco, USA.

5. Lopez F. and Garcia M. (2001), Mean flow and turbulence structure of open-channel flow through non-emergent vegetation, Journal of Hydraulic Engineering, 127, 392-402.

6. Meijer D.G. and Van Velzen E.H. (1999), Prototype-scale flume experiments on hydraulic roughness of submerged vegetation, 28th IAHR Congress, August 22-27 $1999 \mathrm{Graz}$, Austria.

7. Papadonikolaki, G. (2011), Mathematical modeling of flow in vegetated rivers, Diploma Thesis, School of Civil Engineering, National Technical University of Athens, Greece (in Greek).

8. Wilson C.A.M.E., Yagci O., Rauch H.-P. and Olsen N.R.B. (2006), 3D numerical modeling of a willow vegetated river/floodplain system, Journal of Hydrology, 327, 13-21. 